\title{
RESPUESTA FUNCIONAL DE PHYTOSEIULUS PERSIMILIS SOBRE OLIGONYCHUS PUNICAE (ACARI: PHYTOSEIIDAE, TETRANYCHIDAE) EN HOJAS DE AGUACATE
}

\section{FUNCTIONAL RESPONSE OF PHYTOSEIULUS PERSIMILIS ON OLIGONYCHUS PUNICAE (ACARI: PHYTOSEIIDAE, TETRANYCHIDAE) IN AVOCADO LEAVES}

\section{Omar GARCíA-Ángel, ${ }^{1}$ Ernesto CERNA-CHÁVEZ, ${ }^{2}$ Luis Alberto AGUIRRE-URIbe, ${ }^{2}$ Yisa María OCHOA-FUENTES, ${ }^{2}$ JULIO CÉSAR CHACÓN-HERNÁNDEZ ${ }^{3}$ y JERóNIMO LANDEROS-FLORES ${ }^{2, *}$}

\author{
${ }^{1}$ Estudiante de Doctorado en Ciencias, Universidad Autónoma Agraria Antonio Narro, Calzada Antonio Narro \# \\ 1923. C.P. 25315. Buenavista, Saltillo; Coahuila, México. \\ ${ }^{2}$ Universidad Autónoma Agraria Antonio Narro, Calzada Antonio Narro \# 1923. C.P. 25315. Buenavista, Saltillo; \\ Coahuila, México. \\ ${ }^{3}$ Instituto de Ecología Aplicada, Universidad Autónoma de Tamaulipas, Av. División del Golfo 356, Col. Libertad \\ Cd. Victoria, Tamaulipas. C.P. 87019. (OGA)<064rcia@gmail.com>; (ECC)<jabaly1@yahoo.com>; (LAAU) \\ <luisaguirreu@yahoo.com.mx>; (YMOF)<yisa8a@yahoo.com>; (JCCH)<july2019@hotmail.com>; (JLF) \\ $<$ jlanflo@uaaan.mx>. \\ * Autor para correspondencia:<jlanflo@uaaan.mx>. \\ Recibido: 30/05/2017; aceptado: 12/09/2017. \\ Editor responsable: Gabriel Villegas.
}

García-Ángel, O., Cerna-Chávez, E., Aguirre-Uribe, L. A., OchoaFuentes, Y. M., Chacón-Hernández, J. C. y Landeros-Flores, J. (2017). Respuesta funcional de Phytoseiulus persimilis sobre Oligonychus punicae (Acari: Phytoseiidae, Tetranychidae) en hojas de aguacate. Acta Zoológica Mexicana (n.s), 33(3), 503507.

RESUMEN. Se evaluó la respuesta funcional de Phytoseiulus persimilis (Athias-Henriot, 1957) sobre diferentes densidades y estados de desarrollo del ácaro del aguacate Oligonychus punicae (Hirst, 1926) bajo condiciones de laboratorio. Los resultados muestran nula depredación en el estado de huevo, mientras que para el resto de los estados, el depredador exhibió una respuesta funcional tipo II. El máximo consumo de $P$. persimilis sobre $O$. punicae fue en promedio de 23.4 y 14.67 para larvas y ninfas respectivamente a una densidad de 64 presas ofrecidas, mientras que el máximo promedio de consumo en hembras adultas de $O$. punicae fue de 6.07 adultos a una densidad de 16 adultos hembra ofrecidos. Los resultados de la depredación muestran que $P$. persimilis puede ser utilizado como un agente de control biológico. Palabras clave: Control biológico; Aguacate; Respuesta Funcional; Oligonychus punicae; Phytoseiulus persimilis.

\section{INTRODUCCIÓN}

El ácaro del aguacate, Oligonychus punicae (Hirst, 1926) es una plaga común y persistente en México, se encuentra en la parte superior de las hojas provocando un bronceado y reducción de la actividad fotosintética (Peña \& Wysoki, 2008) y año con año se presenta en altas poblaciones
García-Ángel, O., Cerna-Chávez, E., Aguirre-Uribe, L. A., OchoaFuentes, Y. M., Chacón-Hernández, J. C., \& Landeros-Flores, J. (2017). Functional Response of Phytoseiulus persimilis on Oligonychus punicae (Acari: Phytoseiidae, Tetranychidae) in Avocado Leaves. Acta Zoológica Mexicana (n.s), 33(3), 503507.

ABSTRACT. Functional response of Phytoseiulus persimilis (AthiasHenriot, 1957) at different densities and developmental stages of the mite of Oligonychus punicae (Hirst, 1926) under laboratory conditions was evaluated. Results show no predation in the egg state, whereas for the other stages, the predator exhibited a type II functional response. Average consumption of $P$. persimilis over $O$. punicae was 23.4 and 14.67 for larvae and nymphs respectively at a density of 64 prey offered, while the maximum average consumption in adult females of $O$. punicae was 6.07 adults at a density of 16 female adults offered. The results of predation obtained, concluded that $P$. persimilis can be used as a biocontrol agent.

Key words: Biological control; Avocado; Functional Response; Oligonychus punicae; Phytoseiulus persimilis.

(McMurtry, 1985). En densidades de 300 ácaros por hoja ocasionan además del bronceado una defoliación parcial, la cual también se puede presentar cuando se encuentran 70 hembras adultas por hoja durante periodos de sequía (Peña \& Wysoki, 2008). El control químico es el método más utilizado para el manejo de ácaros en México (Soto 2013); sin embargo, mediante el empleo de ácaros depre- 
dadores puede ser otra estrategia adecuada (McMurtry \& Johnson, 1962; McMurtry, 1992; Zhang, 2003). En este sentido Phytoseiulus persimilis (Athias-Henriot, 1957) es un ácaro depredador clasificado como un especialista en Tetranychidae (McMurtry \& Croft, 1997; Croft et al., 2004; McMurtry et al., 2013) y que ha sido empleado en diversos cultivos (Skirvin \& Fenlon, 2001; Kim, 2001; Blindeman \& Van Labeke, 2003; Parvin \& Haque, 2008). En esta investigación se determinó la eficiencia de Phytoseiulus persimilis mediante la respuesta funcional en Oligonychus punicae sobre hojas de aguacate variedad Hass.

\section{MATERIALES Y MÉTODOS}

Se estableció una colonia madre de Oligonychus punicae proveniente de hojas de árboles de aguacate variedad Hass, localizados en Tepic Nayarit $\left(21^{\circ} 26^{\prime} 02.4^{\prime \prime} \mathrm{N}\right.$ $\left.104^{\circ} 54^{\prime} 15.4^{\prime \prime} \mathrm{W}\right)$, estos se multiplicaron bajo condiciones de laboratorio a una temperatura de $26 \pm 2{ }^{\circ} \mathrm{C}$, humedad relativa de $70 \pm 10 \%$ y fotoperiodo de 12:12 luz: oscuridad. El ácaro depredador $P$. persimilis se obtuvo del producto comercial Spidex ${ }^{\circledR}$ (Koppert México, S.A. de C.V.) y multiplicado en hojas de frijol (Phaseolus vulgaris L.) infestadas con Tetranychus urticae (Koch, 1836) (Acari: Tetranychidae).

Los experimentos de respuesta funcional se desarrollaron en una cámara bioclimática (Lab-Line Biotronette Mark III Environmental Chamber. Modelo: 846) bajo las mismas condiciones de desarrollo de la colonia madre, siguiendo la metodología de Brodeur \& Cloutier (1992) con algunas modificaciones. La unidad experimental consistió de un disco de hoja de aguacate de $3 \mathrm{~cm}$ de diámetro el cual se colocó sobre agar solidificado dentro de un recipiente de plástico con capacidad de $30 \mathrm{~mL}$ y una tapa semihermética con un orificio central, cubierto con tela fina. Para el bioensayo de depredación sobre huevos de $O$. punicae se colocaron de 10 a 20 hembras grávidas del ácaro en un periodo de 24 horas, posteriormente las hembras fueron removidas y se ajustó el número de huevos ovipositados a 1, 2, 4, 8, 16, 32 y 64 por cada disco de hoja y 15 repeticiones por densidad, posteriormente se le coloco una hembra adulta de $P$. persimilis en cada densidad de presa. Para los otros estados de desarrollo se siguió el mismo método descrito anteriormente con la excepción de que los huevos se mantuvieron hasta su eclosión y se les permitió el desarrollo hasta los estados correspondientes a evaluar (larvas, ninfas, y hembras adultas). Se registró la depredación a las 24 horas, se determinó el tipo de respuesta funcional (forma de la curva), mediante un análisis de regresión logística, ajustando una ecuación polinomial de la proporción de presas consumidas $\left(N_{a} / N_{o}\right)$ vs el número de presas ofrecidas $\left(\mathrm{N}_{\mathrm{o}}\right)$ (Trexler et al., 1988):

$$
\frac{N_{a}}{N_{o}}=\frac{\operatorname{Exp}\left(P_{0}+P_{1} N_{1}+P_{2} N_{o}^{2}+P_{3} N_{o}^{3}\right)}{1+\operatorname{Exp}\left(P_{0}+P_{1} N_{1}+P_{2} N_{o}^{2}+P_{3} N_{o}^{3}\right)}
$$

Donde: $N_{a}=$ número de presas consumidas, $N_{o}=$ número de presas ofrecidas. $P_{0}, P_{1}, P_{2}$, y $P_{3}=$ parámetros a ser estimados.

Las regresiones se realizaron iniciando con el modelo cúbico y los coeficientes de mayor orden que no fueron significativamente diferentes de cero se eliminaron, hasta que todos los coeficientes que permanecieron en el modelo fueran significativamente diferentes de cero. El símbolo del parámetro lineal $P_{1}$ se usó para distinguir entre la respuesta funcional tipo II y tipo III, Si $P_{1}>0$, la proporción de presas consumidas positivamente dependiente de la densidad inicial, describiendo así una respuesta funcional tipo III. Si $P_{1}<0$, la proporción de presas consumidas disminuye con el número inicial de presas ofrecidas, describiendo así una respuesta funcional de tipo II (Juliano, 2001).

Después de conocer el tipo de respuesta funcional, se estimaron los parámetros de la respuesta funcional: tiempo de manipulación (Th) y capacidad de ataque $\left(a^{\prime}\right)$ de acuerdo al modelo de respuesta funcional tipo II de Holling (1966).

$$
N_{a}=\frac{a^{\prime} N_{o} T}{1+a^{\prime} N T_{h}}
$$

En donde: $N_{a}=$ Número de presas consumidas, $a^{\prime}=$ Tasa de ataque constante (tasa de búsqueda instantánea), $N_{o}=$ Densidad de la presa, $T=$ Tiempo total disponible (24 h en este experimento) y $T_{h}=$ Tiempo de manipulación. Los modelos matemáticos se obtuvieron mediante el procedimiento PROC NLIN de SAS/STAT (SAS 2008).

\section{RESULTADOS Y DISCUSIÓN}

En el Cuadro 1 se presentan los resultados de depredación registrados en los diferentes estados de desarrollo de $O$. punicae. A la densidad de 64 presas, se observó un consumo promedio de 23.4 y 14.67 larvas y ninfas respec- 
Cuadro 1. Número de larvas, ninfas y hembras adultas de Oligonychus punicae consumidos por Phytoseiulus persimilis sobre hojas de aguacate. $N_{0}=$ Presas atacadas observadas; DS = Desviación estándar; Na = Estimados Holling.

\begin{tabular}{ccccccc}
\hline $\begin{array}{c}\text { Densidad inicial } \\
\text { de presas }\end{array}$ & \multicolumn{2}{c}{ Larvas } & \multicolumn{2}{c}{ Ninfas } & \multicolumn{2}{c}{ Adultos } \\
\cline { 2 - 7 } & $\mathrm{No} \pm \mathrm{DS}$ & $\mathrm{Na}$ & $\mathrm{No} \pm \mathrm{DS}$ & $\mathrm{Na}$ & $\mathrm{No} \pm \mathrm{DS}$ & $\mathrm{Na}$ \\
\hline 1 & $1 \pm 0$ & 1.08 & $1 \pm 0$ & 1.10 & $1 \pm 0$ & 1.07 \\
2 & $2 \pm 0$ & 2.11 & $2 \pm 0$ & 2.10 & $2 \pm 0$ & 1.95 \\
4 & $4 \pm 0$ & 4.03 & $4 \pm 0$ & 3.82 & $3.53 \pm 0.64$ & 3.31 \\
8 & $8 \pm 0$ & 7.40 & $7.80 \pm 0.56$ & 6.48 & $5.60 \pm 1.55$ & 5.09 \\
16 & $15.80 \pm 0.56$ & 12.72 & $10.40 \pm 3.36$ & 9.95 & $6.07 \pm 1.39$ & 6.97 \\
32 & $19.07 \pm 4.70$ & 19.84 & $12.33 \pm 2.53$ & 13.59 & & \\
64 & $23.40 \pm 5.33$ & 27.56 & $14.67 \pm 2.82$ & 16.15 & & \\
$\mathrm{a}$ & 1.527 & & 1.452 & & & \\
$\mathrm{Th}$ & 0.0317 & & 0.0556 & & 0.1147 & \\
$\mathrm{X}^{2}$ & 1.47 & & 0.49 & & 0.19 & \\
\hline
\end{tabular}

tivamente, además $P$. persimilis consumió en promedio 6.07 adultos a una densidad de 16 presas ofrecidas y no se observó depredación en el estado de huevo. Al respecto Chant (1961) y McMurtry et al. (1970) mencionan que $P$. persimilis se desarrolla preferentemente sobre presas del género Tetranychus en donde se ha observado depredando en todos los estados de desarrollo, en este sentido, Mushtak \& Nikolay (2015) registraron una depredación de 22.8 huevos de T. urticae en un periodo de ocho horas. En la depredación de larvas ninfas y adultos, el análisis de regresión logística mostró un coeficiente lineal con valores de $\mathrm{P}<0$ (Cuadro 2), lo que sugiere que la proporción de presas muertas disminuyó en función de la densidad inicial de presas ofrecidas, demostrándose una respuesta funcional tipo II (Fig. 1). La respuesta funcional obtenida (Tipo II) concuerda con los registrados por Skirvin \& Fenlon (2003), Xiao \& Fadamiro (2010) y Seiedy et al. (2012), quienes mencionan que $P$. persimilis se ha uti- lizado como agente de control biológico mostrando una respuesta funcional de Tipo II. Parvin et al. (2010) registraron una tasa de consumo de 42.13, 39.6 y 36.9 , para huevos, inmaduros y adultos de $T$. urticae por día respectivamente, a una densidad de 64 individuos por hoja de frijol con preferencia por el estado de huevo, mientras que Naher et al. (2005) señalan un consumo diario de 27.54, 18.12 y 11.32 para los mismos estados de desarrollo respectivamente. En cuanto a los valores de tasa de ataque $\left(a^{\prime}\right)$ en el presente estudio se registraron valores de 1.527 , 1.452 y 1.489 para larvas, ninfas y adultos respectivamente. El tiempo de manipulación del depredador (Th) fue de $0.0317,0.0556$ y 0.1147 para larvas ninfas y adultos respectivamente. En este sentido, Xue et al. (2009) mencionan un promedio diario de consumo de 5.45 adultos por depredador de $P$. persimilis en una densidad de 64 hembras de T. urticae ofrecidas en discos de hoja de frijol y un (Th) de 0.1734 y $\left(a^{\prime}\right)$ de 1.5573. Gharekhani et al. (2014)

Cuadro 2. Resultados del análisis de regresión logística de la proporción de presas de Oligonychus punicae consumidas por Phytoseiulus persimilis. Parámetros seguidos por * son significativos en $p<0,05$, ${ }^{\dagger}$ Valores en paréntesis indican intervalo de confianza al $95 \%$ ambos basados en la fórmula de Wald (SAS, 2008).

\begin{tabular}{|c|c|c|c|}
\hline \multirow[t]{2}{*}{ Estado } & \multicolumn{3}{|c|}{ Parámetros } \\
\hline & Intercepto $\left(\mathrm{P}_{0}\right)$ & Lineal $\left(\mathrm{P}_{1}\right)$ & Cuadrático $\left(\mathrm{P}_{2}\right)$ \\
\hline Huevo & - & - & - \\
\hline Ninfa & $\begin{array}{c}4.0998^{*} \\
(3.4285,4.7710)\end{array}$ & $\begin{array}{c}-0.2404 * \\
(-0.2889,-0.1920)\end{array}$ & $\begin{array}{c}0.00284^{*} \\
(0.0021,0.0036)\end{array}$ \\
\hline
\end{tabular}




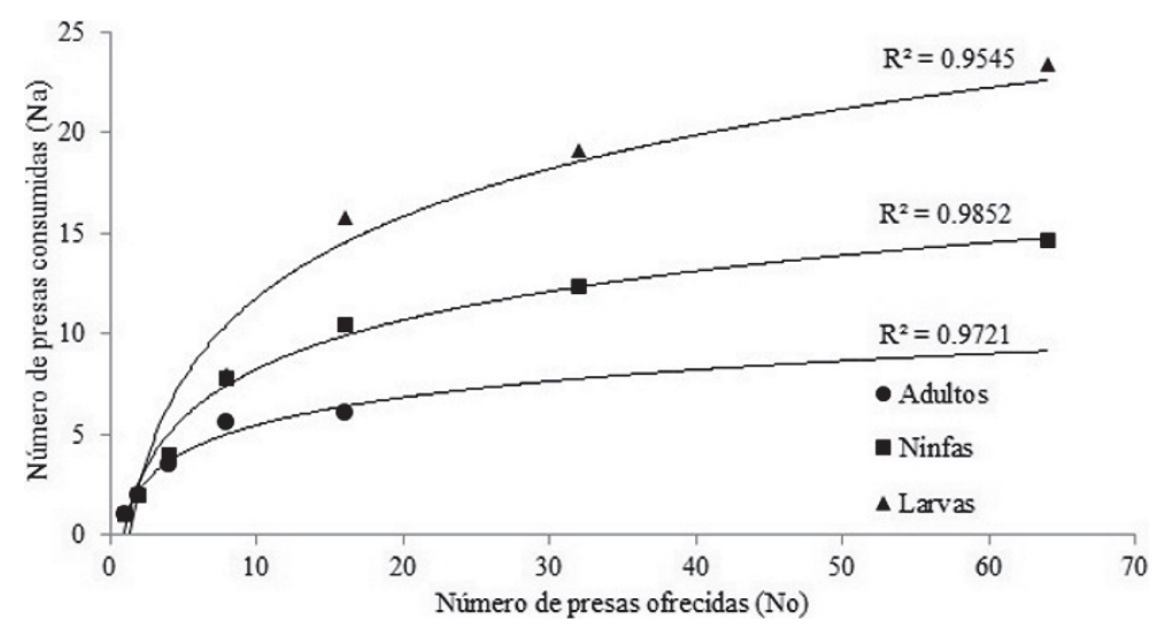

Figura 1. Respuesta funcional de Phytoseiulus persimilis sobre diferentes estados y densidades de Oligonychus punicae.

registran una tasa de ataque y tiempo de manipulación de 2.415 y 0.00154 para estos mismos especímenes. Los valores de $X^{2}$ obtenidos en todos los estados de desarrollo fueron menores al $P<0.05$ lo que indica que se ajustan al modelo matemático tipo II de Holling.

\section{CONCLUSIÓN}

Aunque estos datos se obtuvieron bajo condiciones de laboratorio, los resultados de depredación obtenidos demuestran que $P$. persimilis puede ser utilizado como un agente de control biológico que ayude en el manejo eficiente de $O$. punicae en el cultivo de aguacate.

\section{LITERATURA CITADA}

Blindeman, L. \& Van Labeke, M. C. (2003). Control of the two-spotted spider mite (Tetranychus urticae Koch) in glasshouse roses. Communications in Agricultural and Applied Biological Sciences, 68, 249-254.

Brodeur, J. \& Cloutier, C. (1992). A modified leaf disk method for rearing predaceous mites (Acarina: Phytoseiidae). Phytoprotection, 73, 69-72.

Chant, D. A. (1961). An experiment in biological control of Tetranychus telarius (L.) (Acarina: Tetranychidae) in a greenhouse using the predacious mite Phytoseiulus persimilis Athias-Henriot (Phytoseiidae). Canadian Entomologist, 93, 437-443.

Croft, B. A., Blackwood, J. J. \& McMurtry, J. A. (2004). Classifying life-style types of phytoseiid mites: diagnostic traits. Experimental and Applied Acarology, 33, 247-260

Gharekhani, G., Bahri, M. S. \&Salek-Ebrahimi, H. (2014). Functional response of predatory mite, Phytoseiulus persimilis Athias-
Henriot (Acari: Phytoseiidae) on Tetranychus urticae (Acari: Tetranychidae) in laboratory condition. Journal of Field Crop Entomology, 4, 59-68.

Holling, C. S. (1966). The functional response of invertebrate predators to prey density. Memoirs of the Entomological Society of Canada, 98, 1-86.

Juliano, S. A. (2001). Nonlinear curve fitting: Predation and functional response curves. Pp. 178-196. In: Scheiner, S.M. \& J. Gurevitch. (Eds.) Design and analysis of ecological experiments. Oxford University Press. $2^{\text {nd }}$ Ed. N.Y.

Kim, Y. H. (2001). Control of two spotted spider mite (Tetranychus urticae) by a predatory mite (Phytoseiulus persimilis). Technical Report, National Institute of Agricultural Science and Technology (NIAST) RDA, Korea, 10-01

McMurtry, J. A. \& Johnson, H. G. (1962). The Avocado Brown Mite In Relation To Its Natural Enemies. California Avocado Society 1962 Yearbook 46, 76-81

McMurtry, J. A. (1985). Control of Tetranychidae in crops: Avocado. Pp. 327-333. In: Spider Mites their biology, natural enemies and control. Vol. 1B. W. Helle \& M. W. Sabelis. (Eds.) Elsevier Science Publishers B.V., Amsterdam.

McMurtry, J. A., Huffaker, C. B. \& Van de Vrie, M. (1970). Ecology of tetranychid mites and their natural enemies: A review. I. Tetranychid mites: their biological characters and the impact of spray practices. Hilgardia, 40, 331-390.

McMurtry, J. A. (1992). The Role of Exotic Natural Enemies in the Biological Control of Insect and Mite Pests of Avocado in California Department of Entomology, University of California, Riverside, CA 92521, USA. Proc. of Second World Avocado Congress. 247-252

McMurtry, J. A. \& Croft, B. A. (1997). Life-styles of phytoseiid mites and their roles in biological control. Annual Review of Entomology, 42, 291-321

McMurtry, J. A., De Moraes, G. J. \& Sourassou, N. F. (2013). Revision of the lifestyles of phytoseiid mites (Acari: Phytoseiidae) and implications for biological control strategies. Systematic and Applied Acarology, 18, 297-320 
Mushtak, T. M. \&Nikolay, D. D. (2015). The Feeding Behavior and Functional Response of the Predatory Mite Phytoseiulus persimilis On Different Developental Stages Of Two-Spotted Spider Mite Tetranychus urticae.Biological Sciences НаучныйжурналКубГАУ, $108,1-12$

Naher, N., Islam, W. \& Haque, M. M. (2005). Predation Of Three Predators On Two-Spotted Spider Mite, Tetranychus urticae Koch (Acari: Tetranychidae) J. Life Earth Science, 1, 1-4

Parvin, M. M. \& Haque, M. M. (2008). Control of two-spotted spider mite, Tetranychus urticae Koch (Acari: Tetranychidae) by predators on potted plants. Univ. j. zool. Rajshahi Univ. 27, 51-54

Parvin, M. M., Ali, M. A. \& Mainul, M. H., (2010). Voracity of Three Predators on Two-Spotted Spider Mite, Tetranychus urticae Koch (Acari: Tetranychidae) and Their Developmental Stages. Research Journal of Agriculture and Biological Sciences, 6, 77-83

Peña, J. \& Wysoki, M., (2008). Plagas en Israel, México, Estados Unidos, Centroamérica y Perú. Plagas del palto en México. Pp303 In: R. Ripa \& P. Larral (Eds) Manejo de Plagas en Paltos Y Cítricos. Instituto de Investigaciones Agropecuarias Ministerio de Agricultura. INIA, Chile

SAS, Institute Inc. (2008). SAS/STAT ${ }^{\circledR} 9.2$ User's Guide. Cary, NC: SAS Institute Inc.

Seiedy, M., Saboori, A., Allahyari, H., Talaei-Hassanloui, R. \& Tork, M. (2012). Functional Response of Phytoseiulus persimilis (Acari: Phytoseiidae) on Untreated and Beauveria bassiana
- Treated Adults of Tetranychus urticae (Acari: Tetranychidae). Journal of Insect Behavior, 25, 543-553

Skirvin, D. J. \& Fenlon, J. S. (2001). Plant species modifies the functional response of Phytoseiulus persimilis (Acari: Phytoseiidae) to Tetranychus urticae (Acari: Tetranychidae): implications for biological control. Bulletin of Entomological Research, 91, 61-70

Skirvin, D. J. \& Fenlon, J. S. (2003). The effect of temperature on the functional response of Phytoseiulus persimilis (Acari: Phytoseiidae). Experimental and Applied Acarology, 31, 37-49.

Soto, G. A. (2013). Manejo alternativo de ácaros plagas. Revista de Ciencias Agrícolas, 30, 34-44.

Trexler, J. C., McCullogh, C. E. \& Travis, J. (1988). How can the functional response best be determined? Oecologia, 76, 206-214.

Xiao, Y. F. \&Fadamiro, H. Y. (2010). Functional responses and prey-stage preferences of three species of predacious mites (Acari: Phytoseiidae) on citrus red mite, Panonychus citri (Acari: Tetranychidae). Biological Control, 53, 345-352

Xue, Y., Meats, A., Andrew, G., Beattie, C., Spooner-Hart, R. \& Herron, G. A. (2009). The influence of sublethal deposits of agricultural mineral oil on the functional and numerical responses of Phytoseiulus persimilis (Acari: Phytoseiidae) to its prey, Tetranychus urticae (Acari: Tetranychidae). Experimental and Applied Acarology, 48, 291-302

Zhang, Z. (2003). Mites of greenhouses: identification, biology and control. Cambridge. USA. CABI publishing, $244 \mathrm{p}$. 